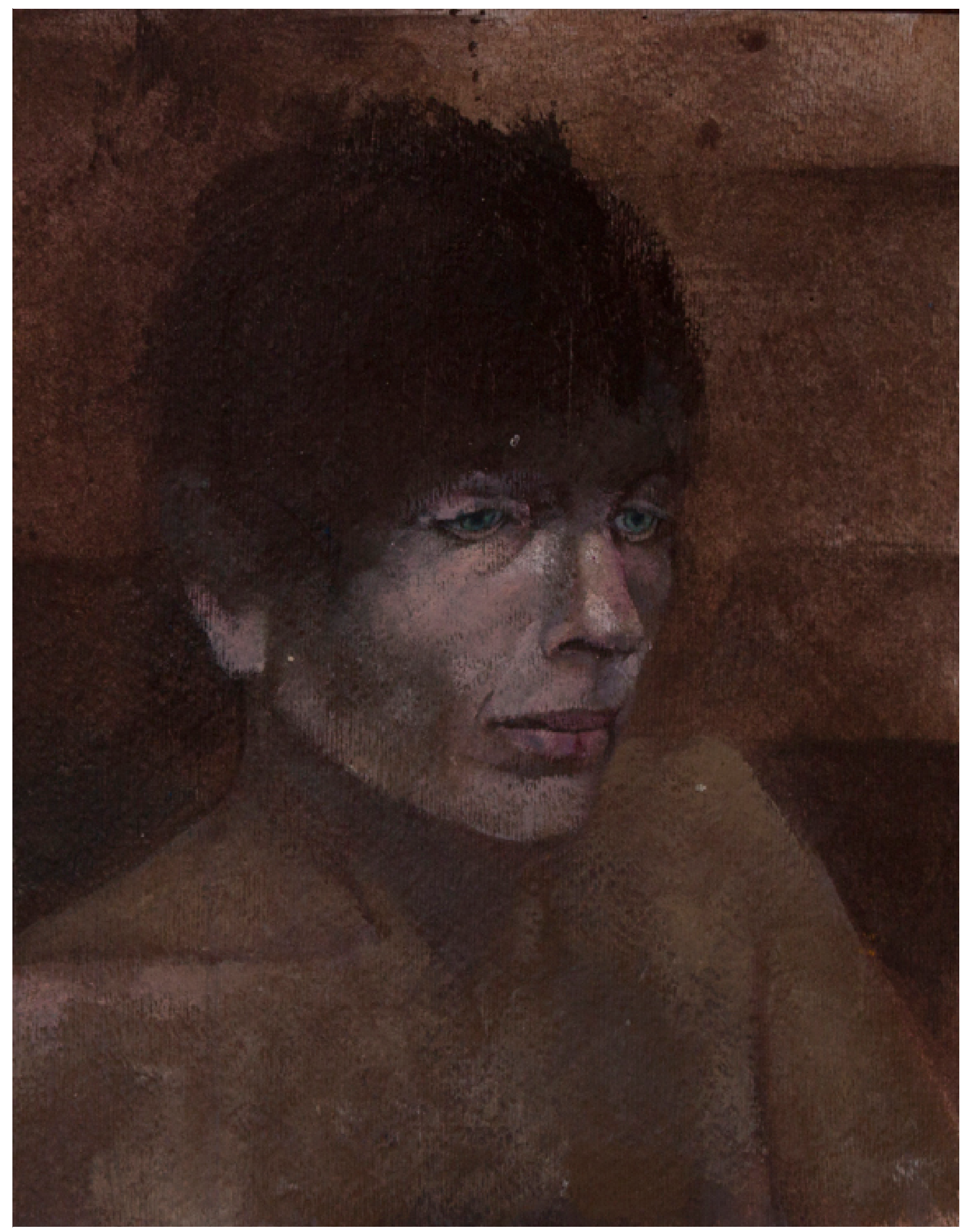




\section{HABILIDADES SOCIOEMOCIONALES EN EL ÁMBITO ESCOLAR DE LOS PLANTELES DE NIVEL MEDIO SUPERIOR DE LA UANL}

\section{SOCIO-EMOTIONAL SKILLS IN THE SCHOOL ENVIRONMENT OF THE UANL HIGH SCHOOL LEVEL SCHOOLS}

RESUMEN

A partir del siglo XX, el manejo de las emociones forma parte importante de la formación integral de los estudiantes en todos los niveles educativos. México no podía ser ajeno a esta tendencia y estableció el Programa Construye- $T$, que tiene como finalidad fomentar habilidades para la vida y se enfoca en tres dimensiones: Conoce T, Relaciona T, Elige T. Este programa, aplicado en la Preparatoria 3 de la UANL, permitió realizar un estudio para determinar si los estudiantes de esta dependencia poseen las habilidades socioemocionales requeridas para establecer relaciones sanas. La finalidad de este programa es contribuir al desarrollo integral de los estudiantes. Este estudio se realizó con estudiantes del sistema escolarizado de cuarto semestre de los tres turnos con que cuenta la escuela. Es importante mencionarlo porque la Preparatoria 3 cuenta modalidades: Abierto, Distancia y Escolarizado y con tres turnos. Este trabajo es una investigación que propone una metodología cuantitativa, descriptiva. Los participantes del estudio fueron seleccionados a partir de un diseño de selección no probabilístico, denominado muestreo por cuotas. Se aplicó un instrumento de evaluación para hacer análisis de frecuencia y tendencia media de las dimensiones del Programa Construye-T. Se realizó un análisis de regresión múltiple lineal para medir el efecto entre dos o más variables independientes sobre una variable dependiente y así determinar cuál variable independiente predice las postulaciones de la investigación.
ABSTRACT

From the twentieth century, the management of emotions has been an important part of the comprehensive training of students at all educational levels. Mexico could not be oblivious to this trend and established the Construye- $T$ Program, which aims to promote life skills and focuses on three dimensions. This program, applied in High School 3 of the UANL, allowed to carry out a study to determine if the students of this unit possess the socio-emotional skills required to establish healthy relationships. The purpose of this program is to contribute to the integral development of the students. This study was conducted with students from the school system in the fourth semester of the three shifts that the school has, it is important to mention it because High School 3 has modalities: Open, Distance and School, and with three shifts. This work is an investigation that proposes a quantitative, descriptive methodology. Study participants were selected from a non-probabilistic selection design, called quota sampling. An evaluation instrument was applied to analyze the frequency and average trend of the dimensions of the Construye-T Program. A linear multiple regression analysis was performed to measure the effect between two or more independent variables on a dependent variable and thus determine which independent variable predicts the research postulations. 
asta muy avanzado el siglo XX, históricamente la educación en todos sus niveles privilegió la adquisición del conocimiento y desarrollo de habilidades cognitivas; sin embargo como resultado de la revolución educativa generada a partir del surgimiento de nuevas teorías en el campo de la psicología y la pedagogía como el constructivismo, y el impacto de éstas sobre los modelos de formación en el aula, se originó un debate sobre la importancia de incluir el manejo de las emociones en la formación integral de los estudiantes.

Un documento referente para el modelo educativo global del siglo XXI y los retos que ésta era representa es La educación encierra un tesoro, Delors (1996); publicado por la UNESCO y preparado por la Comisión Internacional sobre la Educación para el siglo XXI, destaca la importancia de la educación emocional como eje vertebrador de la convivencia, estrechamente vinculada a la salud mental y a la calidad de vida.

La educación emocional se proyecta como un aspecto imprescindible para afrontar los profundos cambios estructurales y sociales que se producen. Repensar la educación desde este enfoque es una utopía necesaria. En este contexto las innovadoras ideas de Peter Salovey y John Mayer (1990) sobre la inteligencia emocional, cubrieron los espacios vacíos dejados por el constructivismo y plantearon que la inteligencia emocional (IE) radica en la capacidad que posee y desarrolla la persona para supervisar tanto sus sentimientos y emociones, como los de los demás, lo que le permite discriminar y utilizar toda esa información para orientar su acción y pensamiento.

Bajo estos referentes, la preocupación por formar personas capaces de adaptarse a las exigencias del mundo se refleja en diversas recomendaciones de organismos internacionales como la UNESCO (1996), que propone 4 pilares para la educación básica:

- Aprender a aprender

- Aprender a hacer

- Aprender a convivir

- Aprender a ser

Las últimas se destacan por su relación con esta investigación.

En México, ya se desarrollan experiencias concretas sobre la educación emocional. En el nivel medio superior el Programa Construye- $T$ representa una tentativa real para darle peso específico al desarrollo de competencias socioemocionales, y señala la tendencia de incluir este aprendizaje mediante programas transversales que están presentes a lo largo del currículo escolar. Sin embargo, existen muy pocos estudios al respecto,

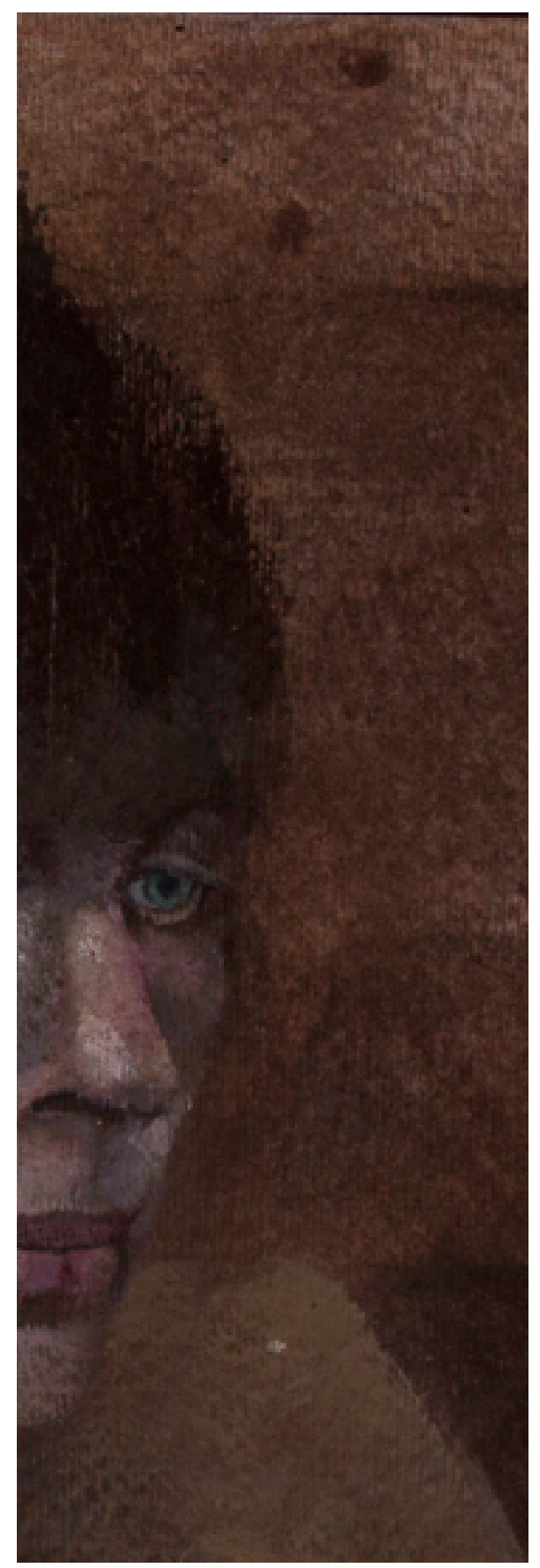

\section{La inteligencia emocional (IE) radica en la capacidad que posee y desarrolla la persona para supervisar tanto sus sentimientos y emociones, como los de los demás, lo que le permite discriminar y utilizar toda esa información para orientar su acción y pensamiento.}




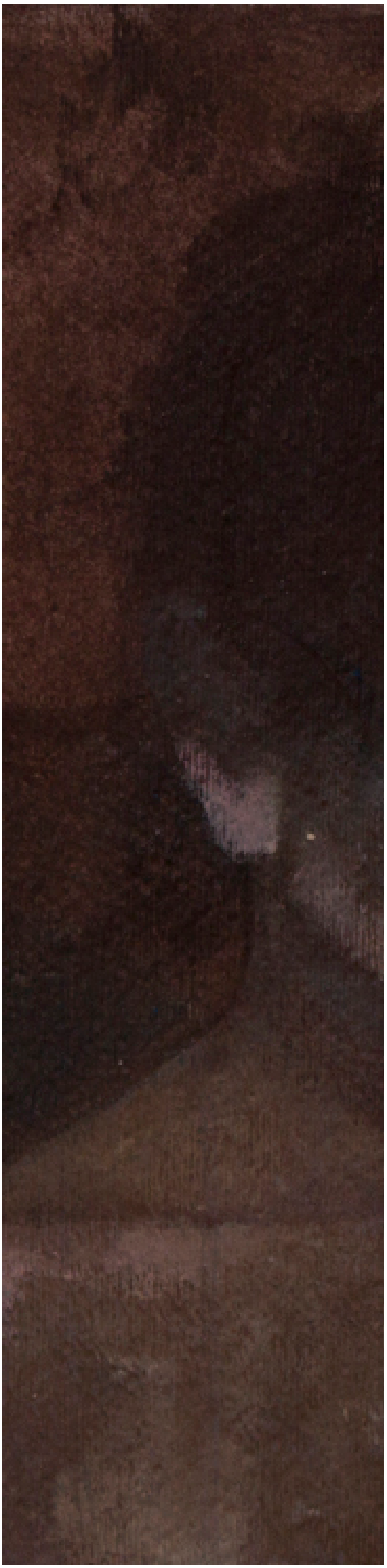

entre ellos destaca una investigación realizada por el mismo programa Construye-T denominada Evaluación de procesos y Seguimiento a Resultados del Programa Construye $T$ (2016), realizado en los siguientes estados: Chihuahua, Estado de México, Morelos y Quintana Roo.

En este contexto, nuestra condición de agentes educativos en el nivel medio superior, particularmente de la Preparatoria No. 3 de la UANL, exige plantear ciertas interrogantes sobre el tema, por ejemplo: ¿Cuál es el estatus emocional de los jóvenes estudiantes de bachillerato?, ¿Cuáles fortalezas y debilidades en este ámbito? ¿Cómo ha impactado el programa Construye $T$ en el desarrollo de sus competencias socioemocionales? Es precisamente la necesidad de responder éstas y otras interrogantes la que da origen al desarrollo de este documento titulado: La condición emocional de los estudiantes de la Preparatoria No. 3 de la Universidad Autónoma de Nuevo León, cuya versión sintética se presenta en esta serie de artículos.

El estudio se realizó en la Preparatoria No. 3 de la UANL, ubicada en el centro de Monterrey, y su objetivo general fue: Realizar un diagnóstico de la condición emocional mediante el estudio de las habilidades socioemocionales de los estudiantes de la Preparatoria No. 3 de la UANL, planteadas por el programa Construye $T$, y determinar su impacto en la formación de los estudiantes.

Este centro escolar brinda servicios educativos a más de 3 mil estudiantes, en diversas modalidades educativas como la presencial y educación a distancia, construyéndose en uno de planteles con mayor población estudiantil en Educación Media Superior del Estado de Nuevo León, México.
Del universo contemplado en la investigación, este trabajo se centró en los alumnos de cuarto semestre como sujetos de estudio por las siguientes 3 razones fundamentales:

- Porque tienen la experiencia de haber participado en el proyecto Construye $T$ desde el inicio del programa.

- $\quad$ Porque se consideró que su madurez psicológica era importante para obtener resultados objetivos.

- Porque dado que cursan la última fase de su trayecto formativo, es importante determinar los logros alcanzados a través del programa en cuanto al desarrollo de competencias socioemocionales.

\section{PLANTEAMIENTO DEL PROBLEMA}

La educación como bien social, no puede limitarse a la búsqueda del éxito personal como meta final del sistema educativo, el planteamiento humanista aborda la cuestión más allá de la función utilitaria que cumple la globalización, la caída de las fronteras nacionales, el surgimiento del libre comercio y el desarrollo económico. Esta visión antropológica tiene consecuencias a la hora de definir aspectos como: el contenido de la enseñanza, las pedagogías, los enfoques educativos, así como la función que corresponde a maestros y otros actores involucrados en la educación. Exige, en suma, un planteamiento abierto y flexible del aprendizaje, que debe extenderse a lo largo de la vida, una propuesta que brinde la oportunidad de realizar su potencial con miras a un futuro razonable y una existencia digna.

La escuela como institución social, está llamada para asumir su parte de responsabilidad en este proceso dirigido al desarrollo integral de los seres humanos que forma, y propiciar dentro de su 


\section{La educación emocional, que tiene por objetivo desarrollar la inteligencia emocional y las habilidades socioemocionales tiene como misión ayudar a las personas a descubrir, conocer y regular sus emociones e incorporarlas como competencias.}

proyecto formativo, el valor añadido de la formación emocional de los alumnos. Delors (UNESCO 1996) reconoce que la educación emocional es un complemento indispensable en el desarrollo cognitivo y una herramienta fundamental de prevención, ya que muchos problemas tienen su origen en el ámbito emocional. La educación emocional, que tiene por objetivo desarrollar la inteligencia emocional y las habilidades socioemocionales tiene como misión ayudar a las personas a descubrir, conocer y regular sus emociones e incorporarlas como competencias.

Uzcategui (1998) define la inteligencia emocional como el conocimiento, luego se transforma en capacidad socio afectiva que facilita al individuo. Ryback (1998), por su parte, afirma que la mejor manera de definir la inteligencia emocional es considerar que se trata de la capacidad de aplicar la conciencia y la sensibilidad para discernir los sentimientos y subyacentes de la comunicación interpersonal, para resistir la tentación que mueve a reaccionar de una manera impulsiva irreflexiva, obrando en vez de ello con receptividad, autenticidad y sinceridad. En este mismo sentido se afirma que puesto que las emociones son involuntarias, las conductas son el producto de las decisiones tomadas por el individuo (Casassus, 2006).

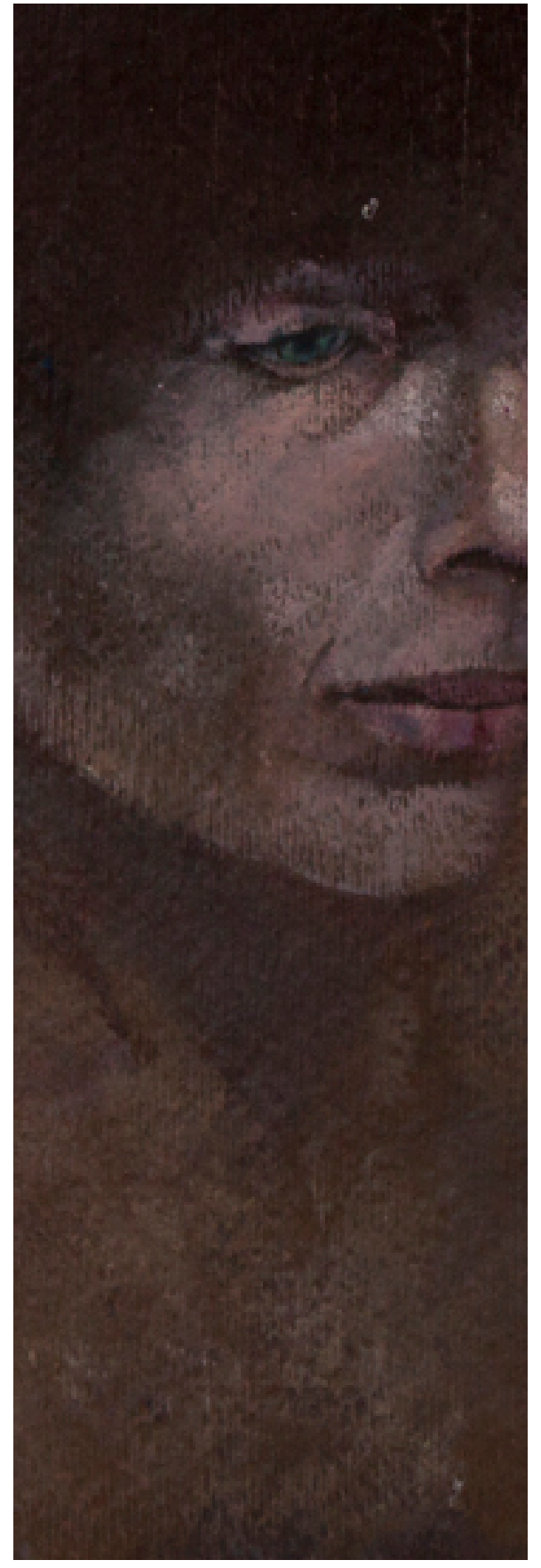

Esto significa que las emociones son eventos o fenómenos de carácter biológico y cognitivo que toman su significación en escenarios sociales. Se pueden clasificar en positivas cuando van acompañadas de sentimientos agradables como la felicidad y el amor; y negativas cuando van acompañadas de sentimientos desagradables, entre las que se encuentran el miedo, la ansiedad, la ira, la hostilidad y la tristeza.

Puesto que las emociones son involuntarias, en tanto las conductas representan el producto de las decisiones tomadas por el individuo, esto supondría que tales conductas son producto de reflexión y pueden ser reafirmadas en el ámbito educacional. Por tanto, la educación emocional pretende dar respuesta a un conjunto de necesidades sociales que no son competentemente atendidas en la educación formal.

Existen múltiples razones para justificar la educación emocional: la implicación de la juventud, lo cual supone un desajuste, como los problemas derivados de baja autoestima, depresión, estrés, violencia, delincuencia, anorexia, SIDA, suicidio, consumo de drogas, conducta sexual no protegida, y otros problemas derivados de las habilidades socioemocionales como fundamento para tomar decisiones. 
Como es sabido, una gran parte de la vida de los estudiantes pasa en los centros escolares, por ello las tendencias en materia educativa del mundo $\mathrm{y}$ en nuestro país empiezan a tomarse en cuenta, particularmente en la enseñanza obligatoria, entre las que se encuentra la educación media superior, programas de habilidades sociales y emocionales, ya que está demostrado que gracias a estos programas, los estudiantes incrementan sus posibilidades de lograr los objetivos relacionados con su salud mental, de ahí el interés por realizar investigaciones, que analiza la condición de los sujetos (estudiantes) y la circunstancias en que se realizan los procesos de su formación.

\section{OBJETIVO GENERAL}

\section{DE LA INVESTIGACIÓN}

El objetivo general de esta investigación es hacer un diagnóstico de la condición emocional mediante el estudio de las habilidades socioemocionales de los estudiantes de la Preparatoria No. 3 de la UANL, planteadas por el programa Construye $T$, como factores que propician su desarrollo personal para la solución de sus problemas en el contexto escolar y de su vida cotidiana.

\section{OBJETIVOS ESPECÍFICOS DE LA INVESTIGACIÓN}

Los objetivos específicos de esta investigación son los siguientes:

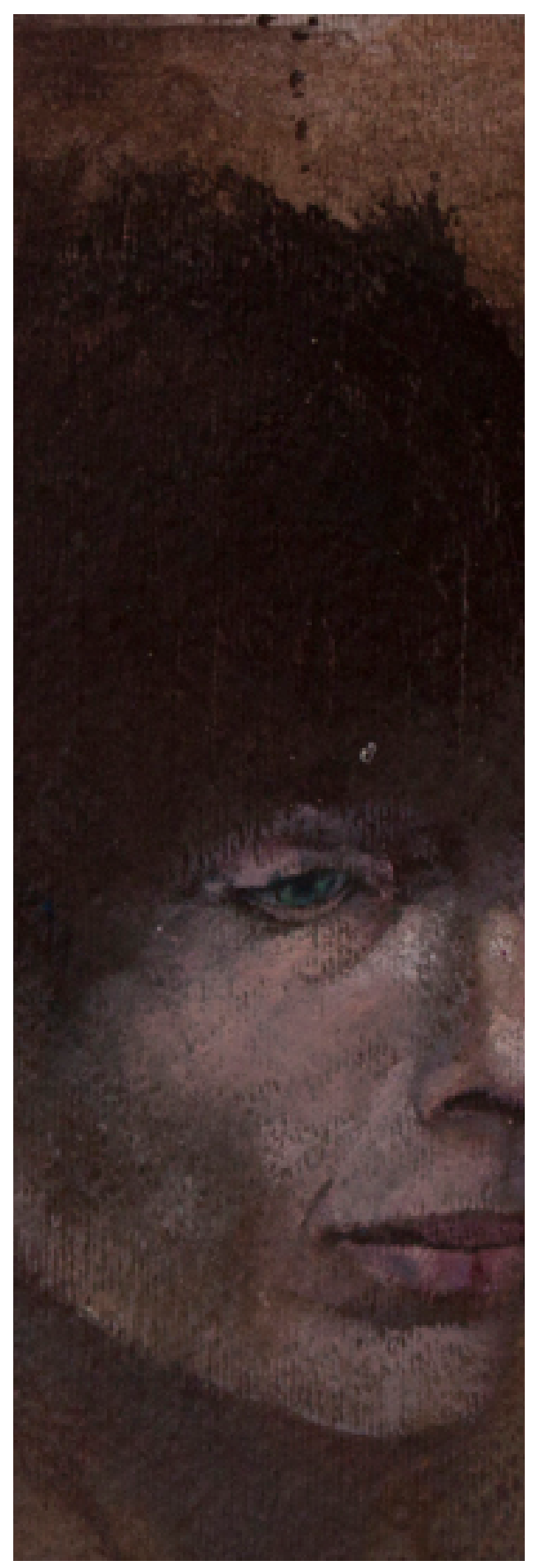

1. Analizar las habilidades socioemocionales de los estudiantes de nivel medio superior de la Preparatoria No. 3 de la UANL, relacionadas con la autoconciencia.

2. Estudiar las habilidades socioemocionales relacionadas con la autorregulación en los estudiantes de Nivel Medio Superior.

3. Analizar si el estudiante posee las habilidades necesarias para dirigir sus esfuerzos en el logro de todas sus metas.

4. Investigar si los estudiantes cuentan con las habilidades emocionales necesarias que le permitan entender a otros y emprender acciones que tengan impacto en el ámbito de su contexto social.

5. Determinar si los estudiantes investigados poseen las habilidades sociales y emocionales requeridas para establecer relaciones sanas con otras personas de su entorno.

6. Analizar si los estudiantes cuentan con las habilidades socioemocionales requeridas para tomar decisiones responsablemente.

\section{HIPÓTESIS}

Los estudiantes de Nivel Medio Superior de la UANL, cuentan con la condición emocional y las habilidades socioemocionales necesarias para actuar positiva y asertivamente frente a los

\section{El objetivo general de esta investigación es hacer un diagnóstico de la condición emocional mediante el estudio de las habilidades socioemocionales de los estudiantes de la Preparatoria No. 3}


riesgos que enfrenta su desarrollo personal y reaccionan emocionalmente de manera adecuada para dar solución a los retos del contexto familiar, escolar y de su vida cotidiana.

\section{JUSTIFICACIÓN}

\section{DE LA INVESTIGACIÓN}

El desarrollo humano tiene como objetivo que las personas logren un progreso personal, social e integral. El objetivo central del desarrollo humano es ampliar las opciones y nivel de bienestar de las personas. El Dr. Juan Lafarga (2013), una autoridad en Desarrollo Humano a nivel mundial, refiere que el desarrollo humano es uno de los conceptos más versátiles de la Psicología Humanista contemporánea y puede ser entendido desde perspectivas antropológicas, individuales y sociales o puede ser abordado como objeto de estudio de la psicología y educación.

Desde hace una década se sabe que el desarrollo humano está profundamente ligado con la educación emocional, una formación que pretende dar respuesta a un conjunto de necesidades sociales que

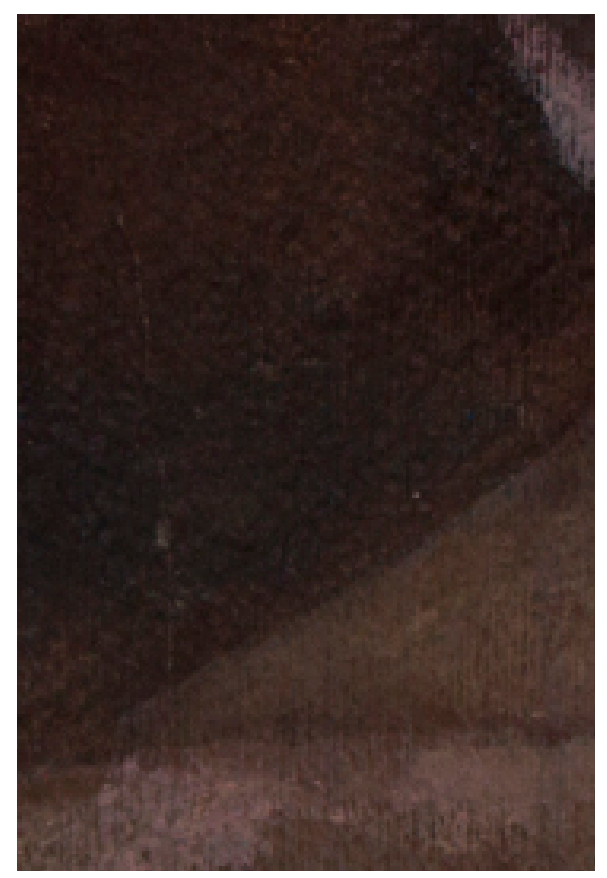

no quedan suficientemente atendidas en la educación formal. Estos argumentos justifican la necesidad de convertir a la educación emocional y particularmente a las habilidades socioemocionales en objeto de estudio.

Entre las iniciativas para incluir en los programas de estudios la educación emocional, particularmente en México se encuentra el programa Construye T; este es un programa de la Reforma Integral que desarrolla la Subsecretaría de Educación Media Superior, lo que demanda no sólo adecuaciones curriculares sino además trabajos de investigación, como el presente, que aporten datos y reflexiones sobre el desarrollo humano de los estudiantes.

Otro aspecto que justifica la realización de esta investigación es que a nivel local son casi inexistentes, estudios metodológicos y de naturaleza científica sobre el tema de los factores que propician el desarrollo humano, la inteligencia emocional y las competencias socioemocionales, por lo que, seguramente el presente documento brindará referentes

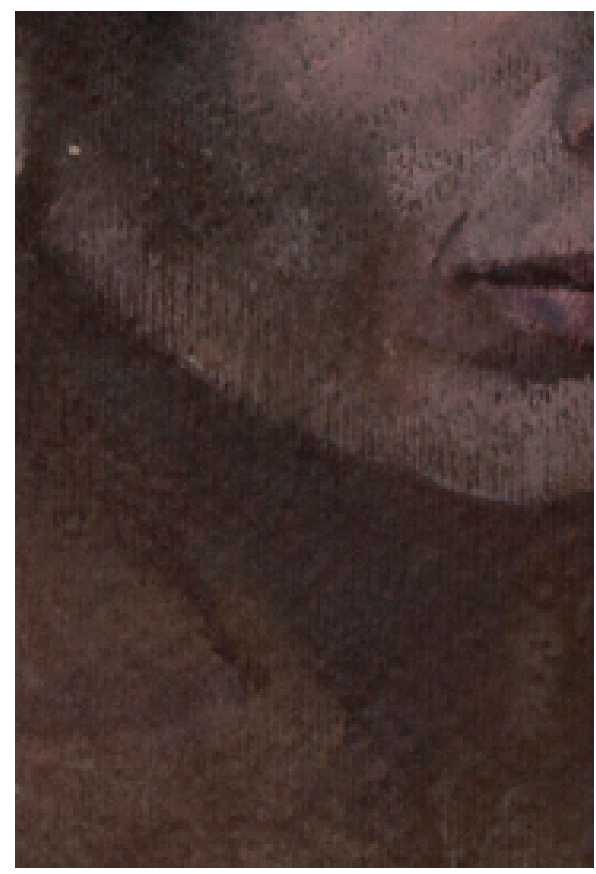

134 importantes para determinar el estatus quo de los estudiantes en el tema estudiado y permitirá generar reflexiones que posteriormente se constituirán en la base de acciones pedagógicas concreta dirigidas a los docentes para fortalecer la formación de sus estudiantes.

Las gestiones escolares, particularmente las encargadas de procurar educación básica, desde Maternal hasta Media Superior, tienen la misión de consolidar las relaciones que se vive dentro de un plantel: entre el docente y los estudiantes, y de los estudiantes entre sí, como una condición ideal para el aprendizaje.

En este marco de referencia, las habilidades socioemocionales o la inteligencia emocional juegan un papel importante y definen a una serie de habilidades que permiten a los estudiantes relacionarse con el entorno (los otros, los demás), aprender activamente y jugar un papel fundamental en entorno familiar, comunitario, laboral y social.

Esta investigación denominada La condición emocional de los estudiantes de

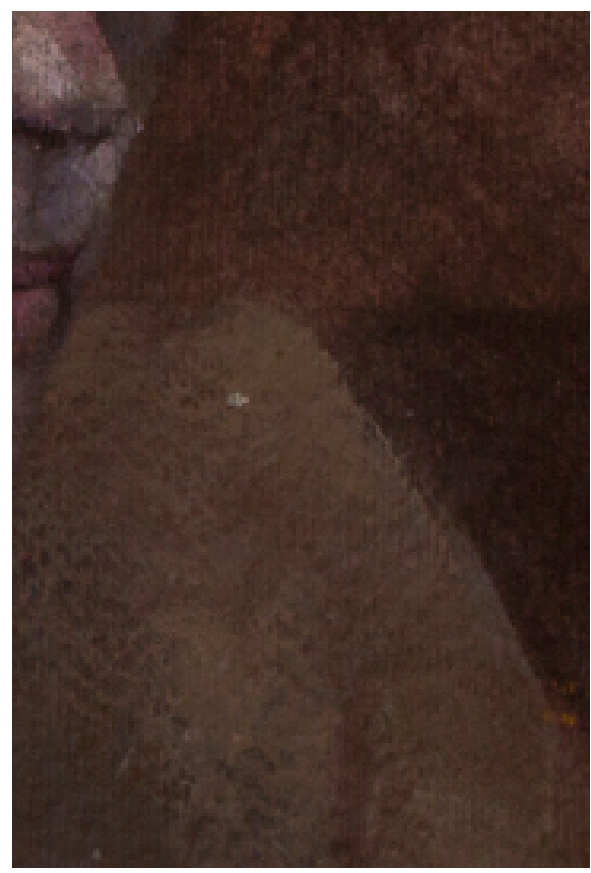




\section{Cuando el aprendizaje y la inteligencia emocional forman parte de la misma ecuación educativa es más probable que los estudiantes utilicen de manera eficiente y responsable lo aprendido.}

la Preparatoria No. 3 de la Universidad Autónoma de Nuevo León. Un estudio de las habilidades socioemocionales propuestas en el programa Construye T para mejorar el ambiente escolar en los planteles del nivel medio superior, muestra que las habilidades socioemocionales de los estudiantes pueden analizarse y establecer una relación entre el ejercicio de tales habilidades con la actuación de los alumnos en el salón de clase y en la escuela, por lo que inciden en el aprendizaje académico.

Cuando el aprendizaje y la inteligencia emocional forman parte de la misma ecuación educativa es más probable que los estudiantes utilicen de manera eficiente y responsable lo aprendido. Se trata de reafirmar la noción de que los estudiantes incorporan a su identidad un sentido de compromiso, responsabilidad, solidaridad y preocupación por el bienestar de sí mismo y de los demás, lo que se traduce en la transformación positiva de su cultura axiológica.

Haciendo una analogía con lo mencionado al inicio de este apartado, puede decirse que el aprendizaje concierne tanto a la cabeza como al corazón, al conocimiento y al sentimiento, a la razón y la emoción, dimensiones de un proceso educativo que se conjugan y dan como resultados estudiantes más inspi-

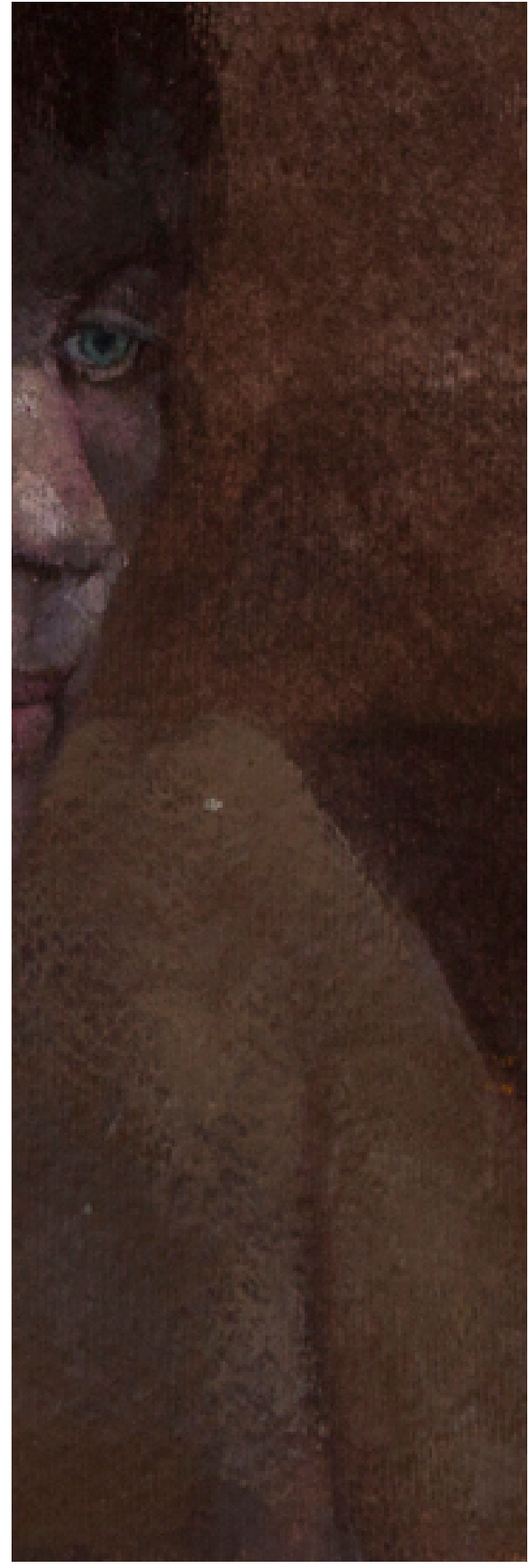

rados, reflexivos y comprometidos con su realidad. La emoción es un concepto que utiliza la Psicología para describir y explicar los efectos producidos por un proceso multidimensional encargado del análisis de situaciones significativas; la interpretación subjetiva de las mismas; la expresión emocional o comunicación de todo el proceso y la preparación para la acción 0 movilización de comportamiento; sin embargo, existen desencuentros para definir el concepto de las emociones.

Desde hace años, como señala Gouk y Hills (2005) existe una falta de acuerdo en su entendimiento y conceptualización; son muchas las preguntas que se han planteado y se siguen planteando científicos y psicólogos acerca de este tema, como, por ejemplo, la frecuente indistinción entre emociones y afectos. Esto ha ido cambiando en los últimos años como resultado del avance en los estudios de las emociones y la conducta.

Para el psicólogo Meyers (2006) las emociones están compuestas por causal fisiológica, comportamiento expresivo y experiencia consciente. Por su parte Bisquerra afirma (2002) que es un estado complejo del organismo caracterizado por una excitación o perturbación que predispone a una respuesta organizada. Las emociones se generan habitualmente 
como respuesta a un acontecimiento externo 0 interno. Fernández-Abascal y Palmero (1999) mencionan:

Una emoción es un proceso que se activa cuando el organismo detecta algún peligro, amenaza 0 desequilibrio con el fin de poner en marcha los recursos a su alcance para controlar la situación.

La emoción se refiere a sentimientos, pensamientos, estados biológicos, estados psicológicos y tipo de tendencias a la acción que los caracteriza. Las emociones son impulsos que nos llevan a actuar, programas de reacción automática con los que nos ha dotado la evolución.

Es importante enfatizar en que cada sujeto experimenta emociones distintas ante un estímulo, esta respuesta emocional depende de sus experiencias anteriores, su aprendizaje y de su circunstancia. Algunas de las reacciones fisiológicas y comportamentales que desencadenan las emociones son innatas, mientras que otras pueden adquirirse. Unas se aprenden por experiencia directa, como el miedo o la ira, pero la mayoría de las veces se aprende por observación de las personas del entorno.

Al igual que ocurre con sus definiciones, también hay diversas propuestas para la clasificación de las emociones, en ellas

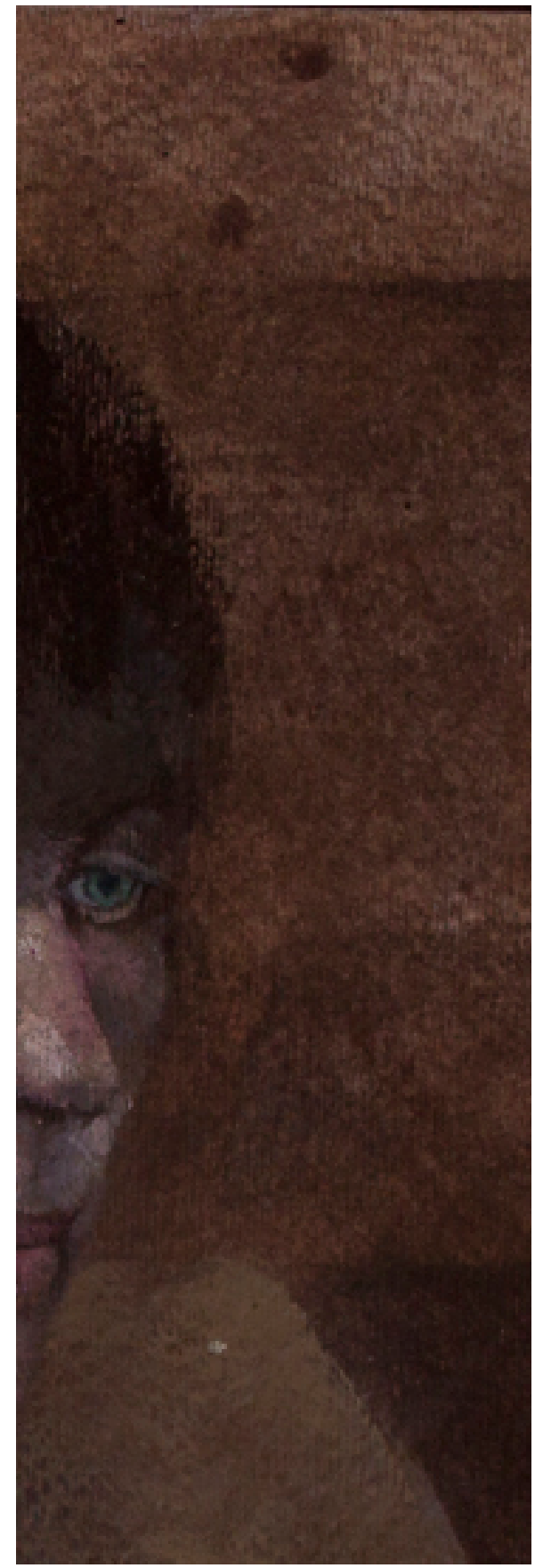

prevalece la sensación de que no existe un acuerdo común; sin embargo, un número importante de autores coinciden en aceptar que existen las emociones básicas y las secundarias. Las básicas pueden variar en número, combinaciones, e intensidad. Por ejemplo, Watson (1919) pensaba que las emociones primarias eran simplemente la ira, el miedo y la alegría, porque se producen de forma espontánea o básica en los niños desde los primeros meses de vida.

Por su parte Del Barrio (2005) añadió a la clasificación anterior otras emociones que, como se puede ver, están relacionadas entre ellas la de la tristeza con carácter básico y contraria a la alegría, la de la sorpresa por su semejanza con la del miedo en sus respuestas o reacciones, en cambio la del interés, no parece tener asociación directa con ninguna de las tres.

Otra categoría es la de las emociones primarias o básicas y secundarias, positivas, negativas y ambiguas. Las positivas son las que motivan el acercamiento al hecho que las produce, las negativas el alejamiento y las ambiguas pueden ser positivas o negativas según las condiciones, y algunas de ellas son la sorpresa, la esperanza, la compasión (Lazarus, 1991). La de Ekman (1992) también es muy globalizada y reconocida por mu-

\section{Del Barrio (2005) añadió a la clasificación anterior otras emociones que, como se puede ver, están relacionadas entre ellas la de la tristeza con carácter básico y contraria a la alegría, la de la sorpresa por su semejanza con la del miedo en sus respuestas o reacciones.}




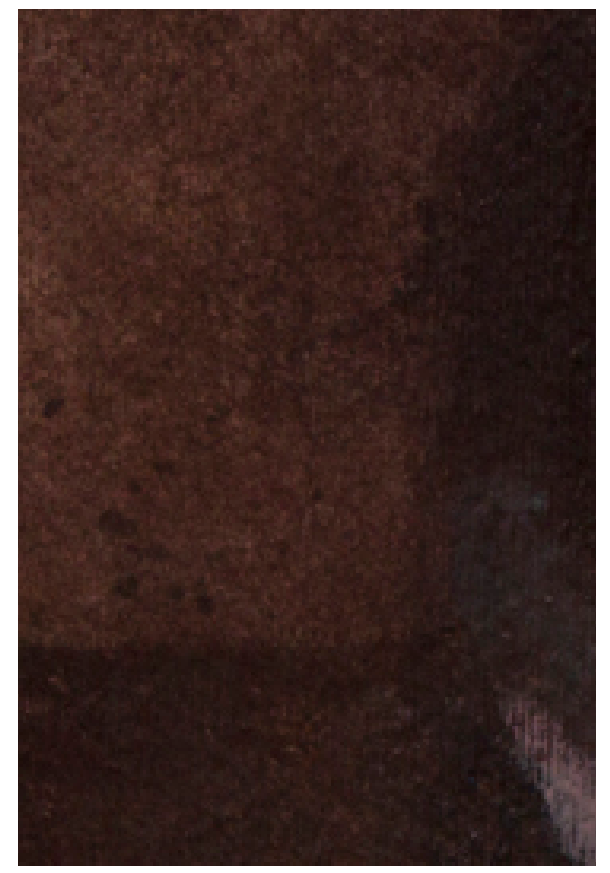

chas culturas y consiste en cuatro tipos de emociones básicas: miedo, ira, tristeza y alegría.

\section{SELECCIÓN DE LA MUESTRA}

La muestra del estudio de investigación fue determinada a partir de un diseño de selección no probabilístico denominado muestreo por cuotas, en el cual la muestra reunida tiene la misma proporción de individuos que toda la población con respecto al fenómeno enfocado, las características o los rasgos conocidos. La distribución de alumnos al momento de la encuesta fue la siguiente: 399 en turno matutino; 337 en turno vespertino; y 77 en turno nocturno; de acuerdo con esta distribución se realizó el muestreo por cuota al $10 \%$, resultando una muestra de: 42 alumnos del turno matutino; 38 del turno vespertino y 10 del turno nocturno, resultando una muestra final de 88 alumnos. Cabe aclarar que la elección de sujetos fue al azar, siguiendo el procedimiento de muestreo aleatorio, seleccionando los números de lista de cada decena terminados en el dígito $2,(2,12$, 22, 32 y 42 en su caso). Los resultados obtenidos no coinciden exactamente con

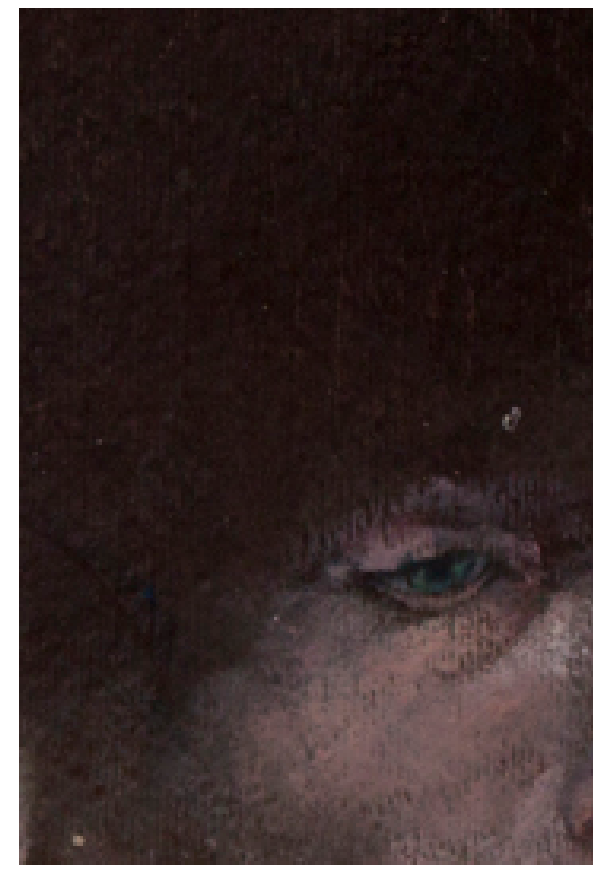

el $10 \%$, sin embargo garantizan una representación porcentual mínima del porcentaje señalado.

\section{CARACTERIZACIÓN \\ DE LA INVESTIGACIÓN Y METODOLOGÍA}

En cuanto a la caracterización de la investigación, ésta se define como descriptiva, documental y no experimental ya que no analiza el comportamiento del objeto de estudio mediante la manipulación de variables para observar los cambios que se producen en los sujetos o fenómenos antes y después de las maniobras de las variables emergentes. Para el desarrollo del presente trabajo se siguió una metodología con un enfoque cuantitativo, ya que se realizó una recolección de información para su análisis mediante mediciones numéricas. Los datos obtenidos se procesaron en el programa SPSS.

\section{INSTRUMENTO DE INVESTIGACIÓN; DISEÑO Y CONSTRUCCIÓN}

El diseño de la encuesta se estructuró a partir de las variables de estudio relacionadas con las competencias socio-

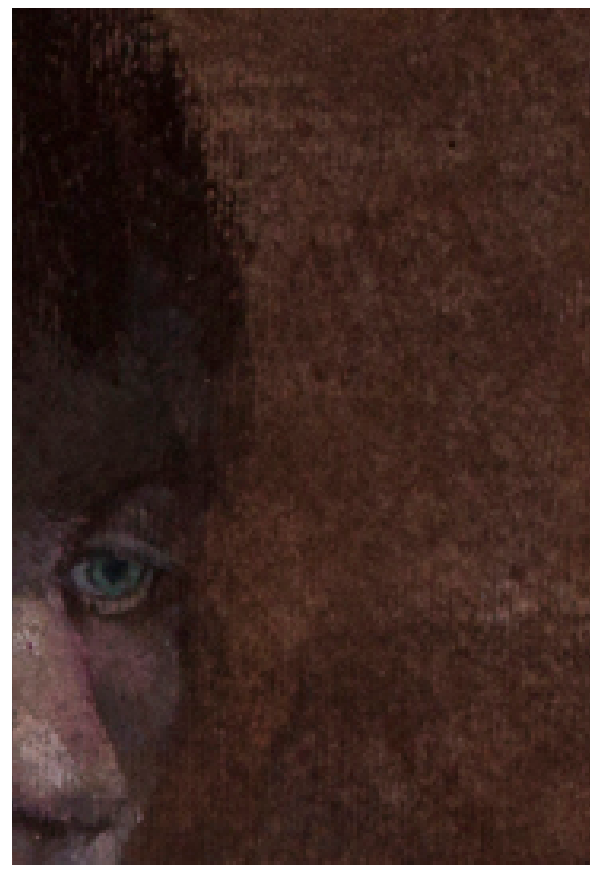

emocionales de los estudiantes de la Preparatoria No. 3 de la UANL. Una vez definidas las dimensiones, variables dependientes e indicadores, se procedió al diseño de ítems. El proceso dio como resultado 76 ítems construidos que se dividieron en 3 apartados de estudio:

1. Del ítem 1 al 25 indagan sobre la dimensión Conoce $t$, que refiere al resultado de un proceso reflexivo mediante el cual un sujeto adquiere noción de su persona, de sus cualidades y características.

2. Del ítem 26 al 55 se explora la dimensión de Relaciona $t$, relacionada con el estado cognitivo a través del cual un sujeto puede interactuar con los estímulos externos que forman la realidad e interpretarlos. Refiere fundamentalmente a la conciencia social propiamente dicha, una conciencia del sí y del entorno que incluye la percepción y conocimiento de los demás integrantes de la comunidad. Cuando se habla de conciencia social, ésta se define como el conocimiento que tienen los individuos sobre el estado de los demás integrantes de su comunidad. 
3. Del ítem 56 al 76 se investigó la dimensión de Elige $t$, este apartado refiere a la capacidad de los sujetos para desarrollar habilidades necesarias para la toma de decisiones reflexivas y responsables en distintos ámbitos para lograr sus metas. La toma de decisiones es el proceso mediante el cual se realiza una elección entre las opciones o formas para resolver diferentes situaciones de la vida en diferentes contextos.

\section{VALIDACIÓN DE LA ENCUESTA}

Para corregir y mejorar el diseño de la encuesta en su primera fase se realizaron 3 procedimientos:

1. La aplicación de una prueba piloto

2. Una validación por especialistas

3. Un análisis de confiabilidad por medio del Alfa de Crombach

\section{PRUEBA PILOTO}

Es una actividad que forma parte del diseño de un cuestionario de investigación. Una vez que el instrumento ha sido diseñado, suele elegirse una pequeña muestra de los casos estudiados, en este caso se eligieron a 10 estudiantes de la muestra antes señalada.

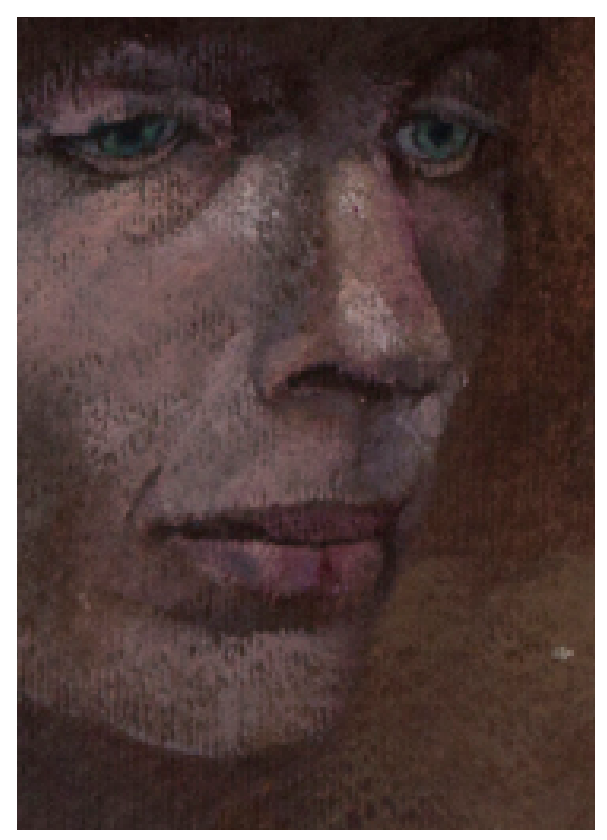

La aplicación de un pre-test o prueba piloto resulta de gran utilidad para el investigador ya que le permite verificar la validez del instrumento, es decir, que éste mida lo que tiene que medir. En la prueba, los investigadores pueden percatarse de si la redacción de las preguntas es adecuada para una buena comprensión; detectar si las preguntas están objetivamente relacionadas con los temas investigados; la extensión del cuestionario adecuado o bien superar otras dificultades que pueden presentarse en el proceso de comunicación.

\section{RESULTADOS}

El instrumento de análisis, se aplicó a 88 estudiantes, de los cuales 36 (40.9\%) pertenecen al género masculino, mientras que 52 (59.1\%) pertenece al femenino. La mayoría de los encuestados se colocó en un rango de edad entre los 15 y 17 años (92\%) mientras que sólo 7 alumnos (8\%) se posicionan en los 180 más. A continuación, se describen los resultados de la encuesta.

Se efectuó un análisis para conocer la consistencia interna del instrumento utilizado. El análisis se aplicó a las 3 variables designadas del estudio mediante el Alfa de Cronbach, éste es un coeficiente que sirve para medir la fiabilidad de una escala de medida y cuya denominación es Alfa. La medida de la fiabilidad mediante el Alfa de Cronbach asume que los ítems medidos en escala tipo Likert miden un mismo constructo y que están altamente correlacionados. Cuanto más cerca se encuentre el valor del alfa a 01 , mayor es la consistencia interna de los ítems analizados y más alto es el grado de confiabilidad.

El análisis de confiabilidad de Cronbach de este instrumento, cuyos resultados se expresan con valor de alfa $\alpha=0.964$, indica una confiabilidad muy alta de los elementos analizados. Ya que el Alpha de Cronbach nos dice en teoría que entre más cerca esté el resultado a 01 mayor es el grado de confiabilidad.

Los alumnos en la pregunta de si les es sencillo ser feliz: $6(6.8 \%)$ respondieron de manera negativa, 20 (22.7\%) marcaron la opción a veces, 29 (33.0\%) dijeron que de manera habitual les resulta sencillo ser feliz, mientras que 33 (37.5\%) no representa ninguna dificultad para ellos el alcanzar la felicidad.

En el caso de si tienen confianza en lo que hacen, los jóvenes externaron sus diferentes puntos de vista: 4 (4.5\%) respondieron que no, ésta fue la respuesta con el menor porcentaje de frecuencia, 30 (34.1\%) marcaron la opción a veces, $25(28.4 \%)$ habitualmente, mientras que 29 (33.0\%) siempre.

También se les cuestionó si cuando enfrentaban un problema lo hacían de manera apropiada: 4 (4.5\%) dijeron que no, siendo esta frecuencia la más baja, 31 (35.2\%) a veces, 31 (35.2\%) habitualmente, y 22 (25.0\%) siempre las manejan de manera apropiada.

A continuación, está la cuestión de si tienen el conocimiento de sus fortalezas y debilidades. Como dato relevante, solamente 1 (1.1\%) dijo no conocerlas, mientras que los demás resultados están dentro de parámetros ordinarios. 21 (23.9\%) respondió a veces, 27 (20.7\%) habitualmente, y 39 (44.3\%) que fue la mayoría, dijeron conocer siempre sus fortalezas y debilidades.

El análisis de regresión múltiple evalúa el efecto entre dos o más variables independientes sobre una variable dependiente, así como predecir el valor de la 
variable dependiente con una o más variables independientes, y estimar cuál es la independiente que mejor predice las puntuaciones de la dependiente. Para este caso, la variable dependiente es:

Considero que tengo las habilidades socioemocionales necesarias para alcanzar las metas que me proponga a lo largo de mi vida.

Y las variables independientes son:

- Autoconocimiento

- Autorregulación

- Conciencia Social

- Colaboración

- Toma Responsable de Decisiones

- Perseverancia

Para determinar cuál o cuáles variables independientes describen a la variable dependiente, se considera aquellas cuyo valor de significancia (Sig.) sea menor a 0.05. Para este análisis se observa que el factor Confianza e identificación de emociones ante un problema, con una significancia de 0.043 describe a la variable dependiente:

Considero que tengo las habilidades socioemocionales necesarias para alcanzar las metas que me proponga a lo largo de mi vida.

\section{CONCLUSIONES}

\section{Dimensión: Conoce-t}

En el análisis de los datos estadísticos de frecuencia, destaca el alto porcentaje de los alumnos que consideran que: siempre están conscientes de su valor como personas. También destaca la actuación responsable de los estudiantes en los distintos ámbitos de su vida, ya que un porcentaje aproximado al $90 \%$ respondió que actúan, habitualmente o siempre, responsablemente. También se puede deducir que los estudiantes se motivan ante las metas y objetivos que se proponen y que un alto porcentaje posee motivación por aprender.

\section{Dimensión: Relaciona-t}

Del análisis de frecuencia de esta dimensión, se puede deducir lo siguiente: A los estudiantes (más de un $80 \%$ ) les gusta ayudar a la gente; respetan las diferentes formas de pensar de las personas (religión e ideología); así mismo, son capaces de llegar a acuerdos con quienes los rodean en beneficio de la integración de un grupo; así mismo, rechaza cualquier forma de discriminación. También destaca que un porcentaje significativo de la población encuestada no comprende la importancia de las personas que toman las decisiones relevantes en una organización, por ejemplo, la escuela $\mathrm{u}$ otras instituciones a las que pertenecen; así mismo manifiestan, en un porcentaje significativo, que no tienen la habilidad de comunicarse eficazmente con los que lo rodean y que manifiestan problemas para expresarse verbal y no verbalmente; acorde con ésta última conclusión, más de una quinta parte de los estudiantes manifestaron que tienen pocas habilidades para relacionarse e interactuar con los demás y que presentan dificultades para trabajar en equipo; en otra conclusión de esta variable, los sujetos encuestados consideran en un porcentaje importante que sus ideas no tienen influencia sobre las personas con las que conviven cotidianamente y no siempre se comportan de acuerdo a las tradiciones de la cultura a la que pertenecen, así mismo, una quinta parte manifestó que es incapaz de asumir el liderazgo de un grupo. Finalmente, se puede resumir que, no obstante, las conclusiones obtenidas en el sentido de las debilidades socioemocionales de los estudiantes para relacionarse con el mundo que les rodea, se destaca que un alto porcentaje sí las posee, y que tales debilidades son producto del desarrollo de una etapa de su vida sumamente compleja, la adolescencia.

\section{Dimensión: Elige-t}

Del índice de frecuencia se deducen las siguientes conclusiones relevantes: los alumnos son capaces de analizar de manera objetiva las ventajas y desventajas al tomar una decisión consciente, así mismo, tienen claras sus metas y objetivos cuando toman una decisión; pueden superar los obstáculos que les dificultan alcanzar sus metas; se esfuerzan lo suficiente para enfrentar el fracaso; son osados para emprender acciones que les ayuden a cumplir sus sueños y consideran que tienen las habilidades socioemocionales necesarias para alcanzar las metas que se proponen a lo largo de su vida. Sin embargo, en esta variable el índice de frecuencia presenta que más de un $30 \%$ de la población encuestada, cuando tiene un problema académico, familiar, sentimental o económico, no siempre lo maneja de manera positiva.

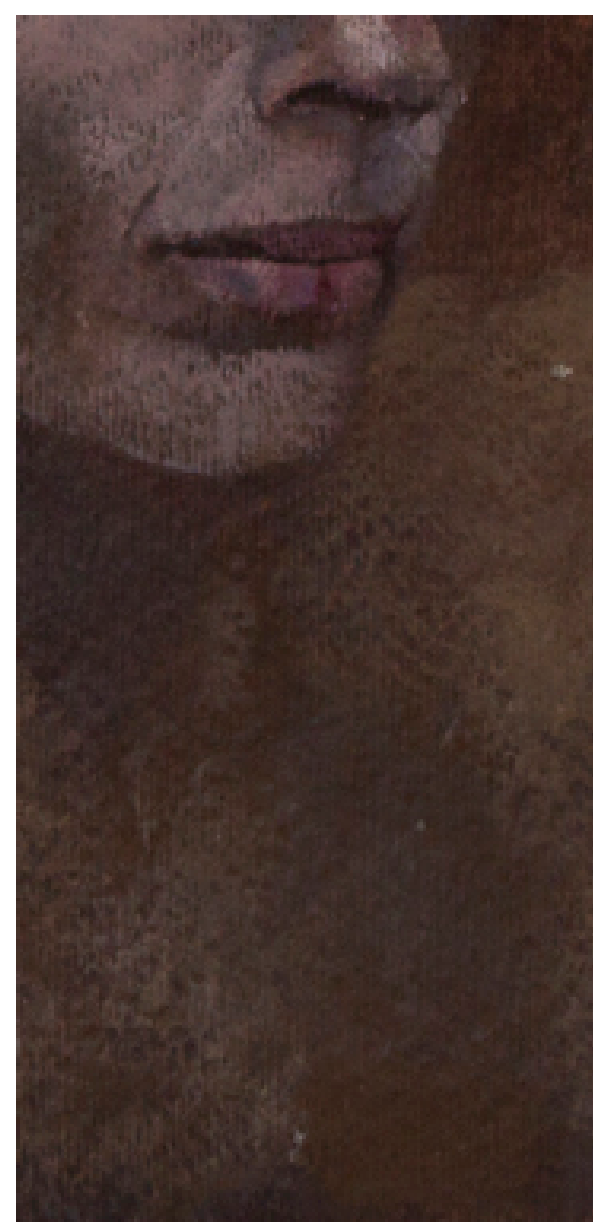




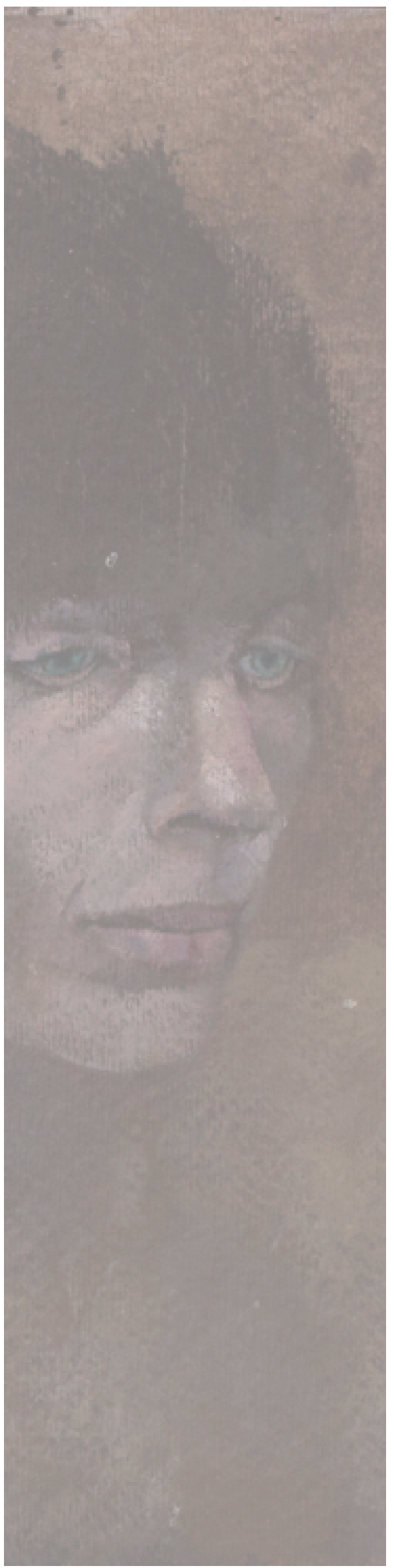

\section{REFERENCIAS}

Bisquerra Alzina, R. (2002). La competencia emocional. Madrid: Síntesis. https://dialnet. unirioja.es/servlet/articulo?codigo $=649432$

Casassus, J. (2006). La educación del ser emocional. Ed. Universidad Virtual del Instituto Tecnológico de Monterrey, México: Ediciones Castillo.

Del Barrio, M.V. (2005). Emociones infantiles. Madrid: Pirámide. Consultado en: https:// www.researchgate.net/publication/31740209 Emociones_infantiles_evolucion_evaluacion_y_prevencion_MV Barrio

Delors, Jacques UNESCO (1996). La educación encierra un tesoro. Informe a la UNESCO de la Comisión Internacional sobre la educación para el siglo XXI. Ed. Grupo Santillana de Ediciones, Madrid.

Ekman (1992). An Argument for Basic Emotions. Cognition and Emotion, 6, 169-200. En Habilidades sociales y emocionales del profesorado de educación infantil relacionadas con la gestión del clima de aula. Consultado en: http://eprints.ucm.es/40450/1/T38117.pdf

Fernández-Abascal y Palmero (1999). Emociones y salud. Ariel. Universidad Nacional de Educación a Distancia (Spain).

Gouk, Penelope; Hills, Helen (eds.) (2005). Representing Emotions: New Connections.

Lafarga, Juan (2013). El desarrollo humano. El crecimiento personal. Universidad Nacional Autónoma de México. Recuperado de: http://www.revistas.unam.mx/index.php/mecedupaz/ article/viewFile/36466/33033

Lazarus, R.S. (1991). Emotion and adaptation. New York: Oxford University Press. Consultado en: http://people.ict.usc.edu/ gratch/CSC1534/Readings/Smith\&Lazarus90.pdf

Meyers David G. (2006). Psicología. Editorial Médica Panamericana. Consultado en https:// psicologiaymente.net/psicologia/psicologia-emocional

Ryback, D. (1998). Trabaje con su Inteligencia, los factores Emocionales al servicio de la Gestión Empresarial y el Liderazgo Efectivo. Editorial EDAF, S.A. España.

Salovey, P. y Mayer, JD (1990). Inteligencia emocional. Imaginación, Cognición y Perso- nalidad, 9, 185-211. Doi: 0.2190 / DUGG-P24E-52WK-6CDG.

UNESCO 1998. Informe Delors. En http://www.unesco.org/education/pdf/DELORS_S.PDF Uzcátegui, I. (1998). El Manual de la Inteligencia Emocional, Emociones Inteligentes: Cómo se puede ser triunfador. Venezuela. Editorial Lithopolar Gráficas.

Watson, J. B. (1919). Psychology: From the standpoint of a behaviorist. Lippincott. En Habilidades sociales y emocionales del profesorado de educación infantil relacionadas con la gestión del clima de aula. Consultado en: http://eprints.ucm.es/40450/1/T38117.pdf 


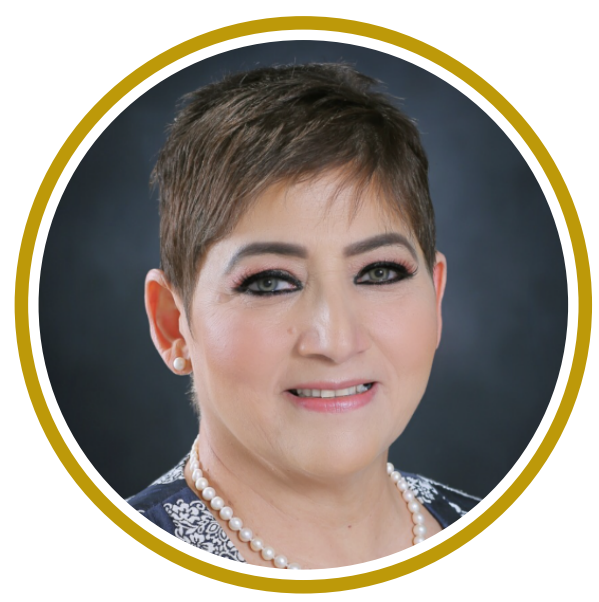

\section{Linda Angélica Osorio Castillo}

Ingeniero Civil por la Universidad Autónoma de Yucatán; maestría en Enseñanza de las Ciencias por la Universidad Autónoma de Nuevo León, Doctorado en Educación por el Instituto Universitario en Sistemas Administrativos de Monterrey. Exdirectora de la Preparatoria 3 de la UANL. Miembro de la Academia Mexicana de la Educación, capítulo Nuevo León, miembro de la Red de Docentes de América Latina y del Caribe. Miembro de la Red de Investigación Educativa de la UANL.

\section{Correo Electrónico:}

lindaaoc@hotmail.com

Recibido: Marzo de 2021

Aceptado: Junio de 2021 


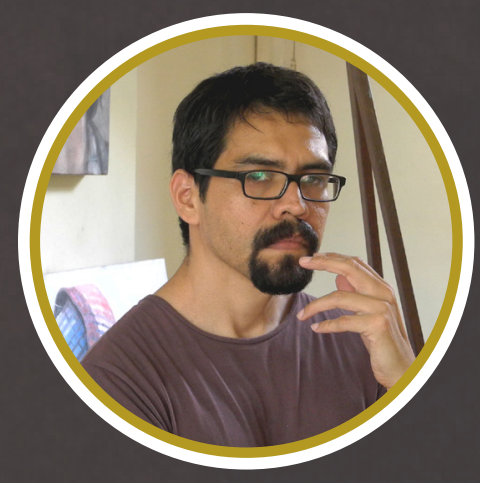

\section{Héctor Manuel Morales Orona}

Egresado de la Facultad de Artes Visuales de la Universidad Autónoma de Nuevo León. Inicia su profesión en el área de las artes gráficas, ilustrando en publicaciones de casas editoriales. Al mismo tiempo alterna esta actividad con la pintura mural. Después de este período inicial, su inquietud creativa se dirige al teatro, donde realiza estudios de arte dramático, cuyo aprendizaje originó nuevas reflexiones sobre el concepto de representación, y la experiencia del juego.

Más adelante, se dedica al estudio de las técnicas antiguas de la pintura haciéndolas su especialidad, y diversifica su profesión como pintor-dibujante y profesor de artes. Desde las técnicas antiguas y la importancia de los materiales, ha encontrado un campo nuevo de resignificaciones conceptuales, representando estas ideas sobre la materia, una fuerte línea de investigación que actualmente trabaja.

Cuenta con múltiples exposiciones colectivas e individuales. Ha participado en ediciones de la Reseña de la Plástica de Nuevo León y su obra se ha expuesto en México, Italia, Cuba y Canadá. 